\title{
EVALUATION OF PRIMARY STABILITY FOR MANDIBULAR IMPLANT OVERDENTURE AFTER OSSEODENSIFICATION USING DENSAH BUR
}

\author{
Nada El Khourazaty* and Ahmed Fahmy**
}

\begin{abstract}
Objectives: To evaluate the effect of osseodensification using Densah bur on the primary stability of mandibular implant overdenture. Materials and Methods: Ten completely edentulous patients were selected and two implants were inserted in the interforaminal region for each patient in a split mouth design; one implant with the conventional drilling system and one implant with the Densah bur drilling system. Implant primary stability was measured using Resonance Frequency Analysis by Osstell immediately after torqueing the implants and every two weeks for 2 months. Results: No significant difference was found regarding Osstell values at all follow up appointments for both groups. Conclusions: comparable results for primary stability with and without osseodensification which still requires further investigation with longer follow up period.
\end{abstract}

KEY WORDS: implants, osseodensification, primary stability.

\section{INTRODUCTION}

The use of implants to retain a mandibular over denture has been recommended as the first treatment choice for the edentulous mandible. From the available evidence; it shows that this treatment leads to a great improvement in both clinical function and patient's reported outcomes when compared to conventional complete dentures.

Successful dental implant placement requires sufficient amount of bone thickness covering the implant so that primary stability is achieved, which is an important requirement for long term success of the implant. ${ }^{1-3}$ From the factors affecting implant primary stability are bone density, surgical protocol, and implant design. ${ }^{4}$ Ample bone thickness covering the implant is not often found as bone resorption after extraction can reach approximately $50 \%$ of the original bone width in under a year. ${ }^{5}$ Also in areas of low bone density maintaining sufficient bone bulk and density is essential to achieve necessary bone to implant contact for obtaining a biomechanically stable implant. ${ }^{6}$

Horizontal bone augmentation for implant placement is often necessary in deficient alveolar

* Lecturer of Removable Prosthodontics, Cairo University

** Associate Professor of Removable Prosthodontics, Cairo University 
ridges. Various methods have been demonstrated in the literature to treat horizontal defects such as guided bone regeneration (GBR), autogenous block grafts, alveolar distraction osteogenesis, ridge splitting, ridge expansion procedures that have been developed and used to augment and treat bone volume defects. ${ }^{7-12}$

Ridge expansion, utilizing motorized rotary expanders, has been suggested as an alternative technique to expand bone by displacing it. Osseodensification is a non-excavating (no cutting) implant site preparation technique. It creates a densified layer of surrounding bone through compaction autografting while simultaneously plastically expanding the bony ridge at the same time. The autografting occurs along the entire length of the osteotomy through a hydrodynamic process with the use of irrigation. The result is a consistently cylindrical and densified osteotomy. ${ }^{13-16}$

The longevity of any implant prosthesis depends on successful osseointegration and implant stability. ${ }^{17,18}$ Consistent osteotomies and densification are important to implant primary stability and to early loading. ${ }^{13}$ One technique for measuring implant stability is resonance frequency analysis aiming to provide an objective measure of implant stability and Osseointegration, which is a noninvasive and does not damage the implant tissue interface. ${ }^{19}$ The resonance frequency analysis technique has been extensively used in experimental and clinical research for the last 10 years.

This study was conducted to investigate the effect of Osseodensification using the Densah bur drilling system on the primary stability in mandibular implants with immediate loading when compared with conventional drilling system.

\section{MATERIALS AND METHODS}

\section{Patient Selection}

Ten completely edentulous male patients were selected from the outpatient clinic of the
Prosthodontics Department; Faculty of Oral and Dental Medicine, Cairo University. The patients'ages ranged from 50-65 years old and were systemically free from any disease that may interfere with dental implant placement and/or osseointegration. Patients also had adequate bone height and width for implant placement, as well as sufficient inter arch space for overdenture construction with normal maxillomandibular relation. Patients also had adequate bone height and width for implant placement (4 to $6 \mathrm{~mm}$ width), as preliminary detected from cone beam computed tomography CBCT confirmed later during pilot drilling. Only cooperative patients capable of following instructions and those with proper neuromuscular coordination were included in the study. Thorough patient history, clinical examination and radiographic assessment were carefully done for verification of the selection criteria. The patients were familiarized with the nature of the study and requested to sign consent forms before beginning the study.

\section{Construction of Complete Dentures}

Study casts were produced from primary alginate impressions for the upper and lower arches of each patient. Acrylic resin special trays were constructed on the diagnostic casts and used in recording the final impressions using zinc oxide and eugenol impression material. Master casts were obtained and occlusion blocks were constructed for jaw relation registration, followed by mounting of the master casts on the articulator. Setting-up of cross linked acrylic resin teeth was done following the lingualized occlusion concept. Try-in was performed, after which the dentures were processed following conventional techniques using high impact acrylic resin.

\section{Implant Placement}

At the delivery appointment, final occlusal adjustments and refinements were done and the denture was delivered to the patients 6 weeks before the surgical appointment to achieve sufficient 
patient adaptation. The finished lower denture was duplicated for each patient and processed in clear acrylic resin in order to construct a surgical guide template to facilitate implant placement during surgery at canine or first premolar area.

After flap reflection, for both osteotomy sites implant manufacturer's pilot drill was used to perform a standard osteotomy of $10 \mathrm{~mm}$ depth. Then the sequential use of Densah Bur (Versah) ${ }^{*} 2.0 \mathrm{~mm}$ pilot, $2.8 \mathrm{~mm}$, and $3.4 \mathrm{~mm}$ multi-fluted tapered burs in a counterclockwise direction (figure 1) under copious irrigation was done in one osteotomy site while drilling the other site using the conventional sequential drilling system. Two $3.6 \mathrm{~mm}$ in diameter, $10 \mathrm{~mm}$ in length implants were placed using the torque wrench in each osteotomy site.**

(Figure 2) Attachment of the smart peg on the implants and the Osstell was used to record the ISQ. Suturing was done after placement of the healing collars.

All patients received 2gm/day amoxicillinclavulanate and $50 \mathrm{mg} / 8$ hours non-steroidal antiinflammatory analgesics for 5 days postoperatively.

Postoperative instructions included a soft diet

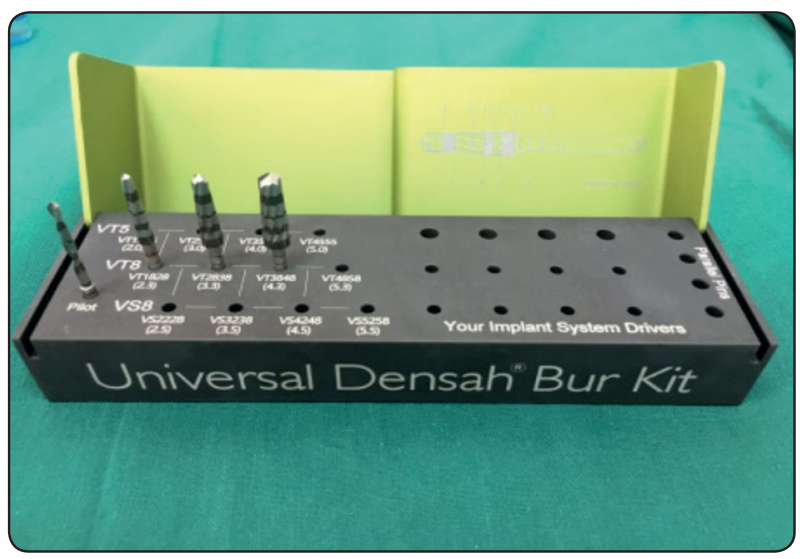

Fig. (1) Universal Densah Bur Kit. and appropriate oral hygiene measures with $0.2 \%$ chlorhexidine mouth rinse.

\section{Overdenture Pick-up}

The dentures were picked up three to five days after surgery for immediate loading protocol. Holes corresponding to the healing collars were drilled in the fitting surface of the denture to allow seating of the denture without any interference with the housings, as proved by absence of rocking, pressure indicating paste and proper occlusion. Soft reline denture material ${ }^{* * *}$ was placed in the relieved areas of the denture and the denture was seated in the patient mouth. The resin was left to polymerize while the patient was closing in centric jaw relation with gentle pressure. The overdenture was removed, trimmed and polished. The dentures were delivered and oral hygiene instructions were given to the patients.

\section{Implant Stability Measurement}

Implant primary stability measurements for each implant was done at the time of surgery and at 2, 4, 6 and 8 weeks. Implant stability was assessed using the Osstell. ${ }^{* * * *}$ The Osstell was used according to the

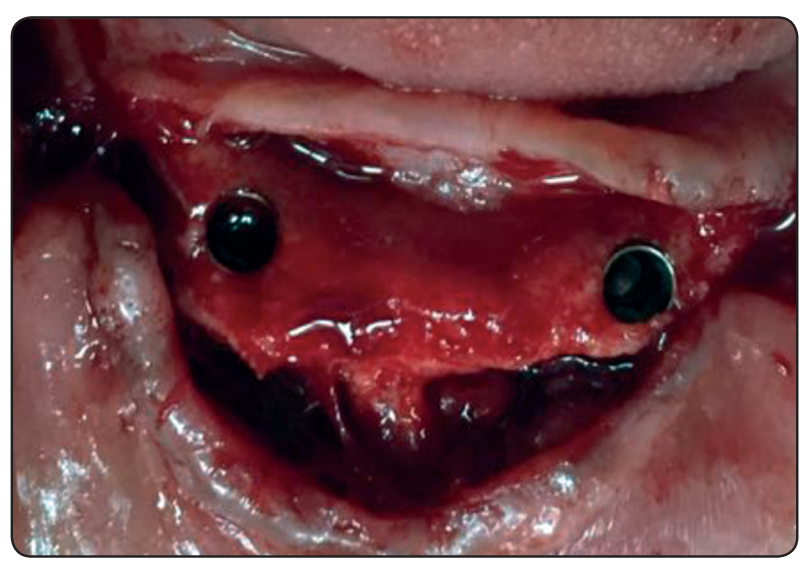

Fig. (2) Implants placed in the osteotomy sites

\footnotetext{
* DENSAH Bur Kit VDBK

** Dentium implant system (Dentium, Samsung-dong, Seoul, Korea)

*** Superline Dentium implant system

**** Dura base soft cushion rebase, Reliance Dental Mfg. Co. warth, Illinois 604B2

***** Siemens AG, Bensheim, Germany
} 
manufacturer's instructions and held perpendicular to the provided implant smart peg. (fig 3) Osstell values (ISQ) were obtained for the buccal, lingual, mesial and distal surfaces of each implant. Three measurements were made for each surface and the mean was obtained for statistical analysis.

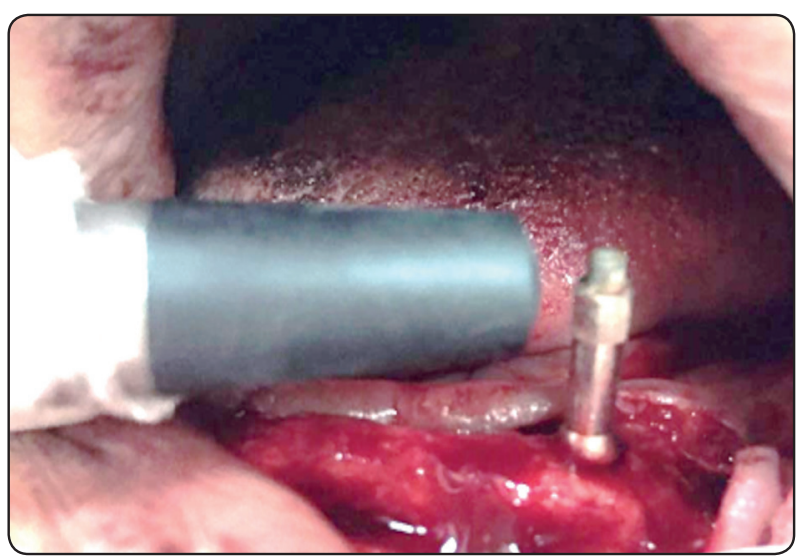

Fig. (3) The Osstell used for implant stability measurements.

\section{Statistical Analysis}

Mean values were obtained for each implant for Osstell values. The mean and standard deviation values were calculated for each group in each test. Data were explored for normality using KolmogorovSmirnov and Shapiro-Wilk tests, data showed parametric (normal) distribution. Independent sample t-test was used to compare between two groups in non-related samples. Repeated measure ANOVA was used to compare between more than two groups in related samples. Paired sample t-test was used to compare between two groups in related samples. (Split mouth technique), Two-way ANOVA was used to test the interaction between variables. The significance level was set at $\mathrm{P} \leq 0.05$. Statistical analysis was performed with IBM ${ }^{\circledR}$ SPSS ${ }^{\circledR}$ Statistics Version 20 for Windows.

\section{RESULTS}

\section{Implant Stability}

The results of this study showed a decrease in ISQ values after implant insertion at 2,4,6 and 8 weeks in both groups. Table (1) These changes were statistically significant at all follow up appointments in the two groups. In group I (densah) the highest mean value of ISQ was $76.00 \pm 6.41$ found at the implant insertion, while the lowest mean value of ISQ was $70.13 \pm 5.22$ found at 6 weeks. As for group II (conventional) the highest mean value of ISQ was $78.88 \pm 7.18$ found at implant insertion, while the lowest mean value of ISQ was $65.88 \pm 15.34$ found at 2 weeks. On comparing the two groups there was no statistically significant difference between (Densah) and (Conventional) groups at 0,2,4,6 and 8 weeks.

Table (1): The mean, standard deviation (SD) values of stability of different groups.

\section{Variables}

\begin{tabular}{cr} 
& Mean \\
$\mathbf{0 w}$ & 76.00 \\
$\mathbf{2 w}$ & 75.13 \\
$\mathbf{4 w}$ & 71.00 \\
$\mathbf{6 w}$ & 70.13 \\
$\mathbf{8 w}$ & 72.88 \\
p-value & \\
\hline
\end{tabular}

\section{Densah}

SD

6.41

6.22

2.56

5.22

4.73

0.013*
Stability

\section{Conventional}

SD

$\begin{array}{cc}\text { Mean } & \text { SD } \\ 78.88 & 7.18 \\ 65.88 & 15.34 \\ 70.38 & 4.37 \\ 74.75 & 2.38 \\ 74.88 & \\ & 0.35 \\ & \text { 0.006* }\end{array}$

0.413ns

0.136ns

0.732ns

0.039*

0.253ns 


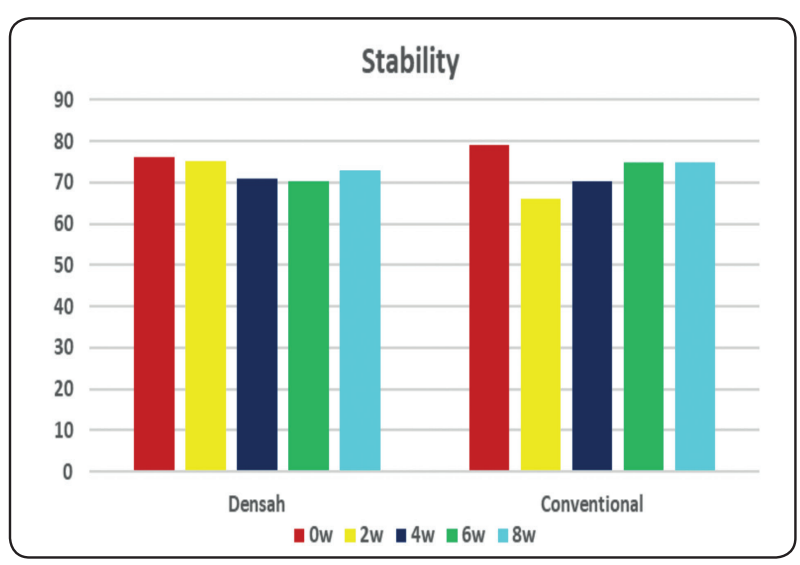

Fig. (4): Bar chart representing stability for different groups

\section{DISCUSSION}

The immediate implant loading approach has been studied extensively since it has been introduced. However, careful planning and case selection are needed to ensure implant stability and success to improve the prosthetic outcomes..$^{20,21}$

The hard and the soft tissue have a significant biological response to immediate implant loading with the highest mechanical stability achieved with threaded implants. ${ }^{13}$ Also implant to bone contact, implant stability and osseointegration are improved by progressive lateral compression during drilling. ${ }^{22}$

The new concept for osteotomy called osseodensification (OD) has been at the forefront of changes in surgical site preparation for implantology. Osseodensification is a surgical instrumentation technique where bone is compacted into open marrow spaces during drilling, increasing implant insertion torque through densification of osteotomy site walls. ${ }^{23}$ It achieves bone expansion at different sites of varying bone densities. This procedure has also shown improvement in achieving better implant primary stability and better osteotomy than conventional implant drills. ${ }^{6}$ In low-density bone, implants presents higher insertion torque levels when placed in osseodensified drilling sites, with no osseointegration impairment compared to standard drilling methods. ${ }^{13}$

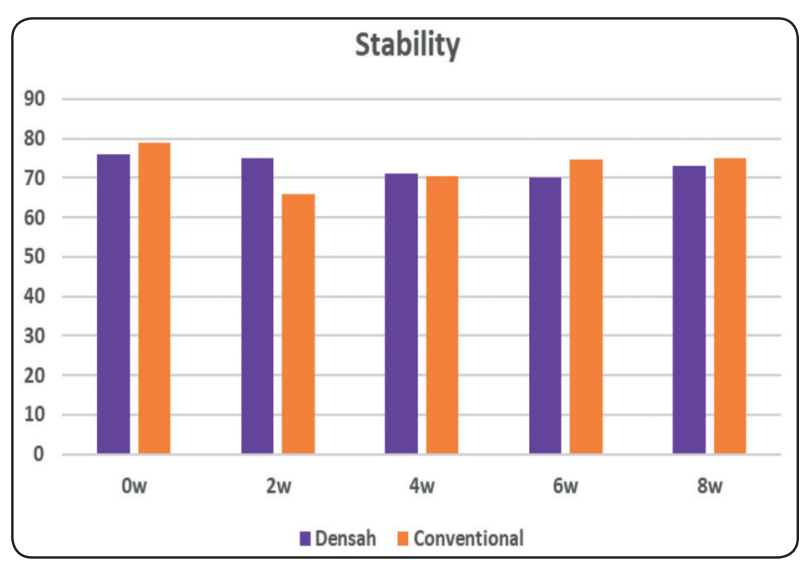

Fig. (5): Bar chart representing stability for different groups

Implant stability is considered one of the important indications of successful osseointegration ${ }^{24}$. The clinical perception of primary implant stability is usually based on the cutting resistance of the implant during insertion. ${ }^{25}$ Resonance frequency analysis (RFA) offers a noninvasive clinical measurement of stability and osseointegration of implants; it is a useful tool to establish implant loading time. The RFA values are represented by a quantitative unit called the Implant Stability Quotient (ISQ) on a scale from 1 to $100 .{ }^{26}$

The results of this study showed a decrease in ISQ values after implant insertion at 2,4,6 and 8 weeks in both groups; which may be attributed to the dome shaped healing abutments which are 2 to 4 $\mathrm{mm}$ in height that provide support and disseminate the lateral forces during osseointegration. Also this can be attributed to the fact that implants are mechanically stable right after insertion with high ISQ values denoting high primary stability and then decline due to the process of bone necrosis that happens after the osteotomy site preparation which is then replaced with new bone formation after week $8 .^{272829}$ However this decrease in stability was statistically insignificant at all appointments. The difference between the two groups at the end of the follow-up period was also found to be insignificant. 
As opposed to previous in vivo and invitro studies that were performed in low density bone and in the maxilla; ${ }^{6,23,30-35}$ the results of this study showed insignificant difference between drilling with Densah bur and conventional burs on implant primary stability; that can be attributed to the area of the performed osteotomy; as the mandibular interforaminal region is composed of dense cancellous bone as opposed to the low density bone found in posterior mandible region. On the other hand Densah bur can play an important role in expanding narrow ridges during osteotomy site prepration, however Osseodensification in areas of dense bone still requires further investigation with longer follow up.

\section{CONCLUSION}

- There is no difference in primary stability of mandibular interforaminal implants when drilling is carried out by Densah bur or conventional bur.

\section{REFERENCES}

1. Marquezan, M., Osório, A., Sant'Anna, E., Souza, M. M. \& Maia, L. Does bone mineral density influence the primary stability of dental implants? A systematic review. Clin. Oral Implants Res. (2012). doi:10.1111/j.16000501.2011.02228.x

2. Ostman, P.-O. et al. Resonance Frequency Analysis Measurements of Implants at Placement Surgery. Int. J. Prosthodont. (2006).

3. Trisi, P., De Benedittis, S., Perfetti, G. \& Berardi, D. Primary stability, insertion torque and bone density of cylindric implant ad modum Branemark: Is there a relationship? An in vitro study. Clin. Oral Implants Res. (2011). doi:10.1111/j.1600-0501.2010.02036.x

4. Turkyilmaz, I., Aksoy, U. \& McGlumphy, E. A. Two alternative surgical techniques for enhancing primary implant stability in the posterior maxilla: A clinical study including bone density, insertion torque, and resonance frequency analysis data. Clin. Implant Dent. Relat. Res. (2008). doi:10.1111/j.1708-8208.2008.00084.x

5. Nedir, R. et al. A 7-year life table analysis from a prospective study on ITI implants with special emphasis on the use of short implants. Results from a private practice. Clin. Oral Implants Res. (2004). doi:10.1111/j.16000501.2004.00978.x

6. Pai, U., Rodrigues, S., Talreja, K. \& Mundathaje, M. Osseodensification - A novel approach in implant dentistry. J. Indian Prosthodont. Soc. (2018). doi:10.4103/ jips.jips_292_17

7. Urban, I. A., Nagursky, H., Lozada, J. L. \& Nagy, K. Horizontal Ridge Augmentation with a Collagen Membrane and a Combination of Particulated Autogenous Bone and Anorganic Bovine Bone-Derived Mineral: A Prospective Case Series in 25 Patients. Int. J. Periodontics Restor. Dent. (2013). doi:10.11607/prd.1407

8. Monje, A. et al. Horizontal Bone Augmentation Using Autogenous Block Grafts and Particulate Xenograft in the Severe Atrophic Maxillary Anterior Ridges: A Cone-Beam Computerized Tomography Case Series. J. Oral Implantol. (2015). doi:10.1563/AAID-JOI-D-13-00219

9. Simion, M., Baldoni, M. \& Zaffe, D. Jawbone enlargement using immediate implant placement associated with a split-crest technique and guided tissue regeneration. Int. J. Periodontics Restorative Dent. (1992).

10. Santagata, M., Guariniello, L. \& Tartaro, G. A Modified Edentulous Ridge Expansion Technique for Immediate Placement of Implants: A Case Report. J. Oral Implantol. (2011). doi:10.1563/AAID-JOI-D-10-00035.1

11. Funaki, K., Takahashi, T. \& Yamuchi, K. Horizontal alveolar ridge augmentation using distraction osteogenesis: comparison with a bone-splitting method in a dog model. Oral Surgery, Oral Med. Oral Pathol. Oral Radiol. Endodontology (2009). doi:10.1016/j.tripleo.2008.10.005

12. Ella, B., Laurentjoye, M., Sedarat, C., Masson, E. \& Rouas, A. Mandibular Ridge Expansion Using a Horizontal Bone-Splitting Technique and Synthetic Bone Substitute: An Alternative to Bone Block Grafting? Int. J. Oral Maxillofac. Implants (2014). doi:10.11607/jomi.2201

13. Lahens, B. et al. Biomechanical and histologic basis of osseodensification drilling for endosteal implant placement in low density bone. An experimental study in sheep. J. Mech. Behav. Biomed. Mater. (2016). doi:10.1016/j. jmbbm.2016.06.007

14. Trisi, P., Berardini, M., Falco, A. \& Podaliri Vulpiani, M. New osseodensification implant site preparation method to increase bone density in low-density bone: In vivo evaluation in sheep. Implant Dent. (2016). doi:10.1097/ ID.0000000000000358 
15. Podaropoulos, L. Increasing the Stability of Dental Implants: the Concept of Osseodensification. Balk. J. Dent. Med. (2017). doi:10.1515/bjdm-2017-0023

16. Tretto, P. H. W., Fabris, V., Cericato, G. O., Sarkis-Onofre, R. \& Bacchi, A. Does the instrument used for the implant site preparation influence the bone-implant interface? A systematic review of clinical and animal studies. International Journal of Oral and Maxillofacial Surgery (2018). doi:10.1016/j.ijom.2018.04.005

17. Sakka, S., Baroudi, K. \& Nassani, M. Z. Factors associated with early and late failure of dental implants. Journal of investigative and clinical dentistry (2012). doi:10.1111/ j.2041-1626.2012.00162.x

18. Oh, T.-J., Yoon, J., Misch, C. E. \& Wang, H.-L. The causes of early implant bone loss: myth or science? J. Periodontol. 73, 322-33 (2002).

19. Sennerby, L. \& Meredith, N. Implant stability measurements using resonance frequency analysis: Biological and biomechanical aspects and clinical implications. Periodontol. 2000 (2008). doi:10.1111/j.1600-0757.2008.00267.x

20. Papaspyridakos, P., Chen, C.-J., Chuang, S.-K. \& Weber, H.-P. Implant Loading Protocols for Edentulous Patients with Fixed Prostheses: A Systematic Review and Meta-Analysis. Int. J. Oral Maxillofac. Implants (2014). doi:10.11607/jomi.2014suppl.g4.3

21. Gill,A.\& Rao,P.Primary stability: The password of implant integration. J. Dent. Implant. (2012). doi:10.4103/09746781.102223

22. Alifarag, A. M. et al. Atemporal osseointegration: Early biomechanical stability through osseodensification. J. Orthop. Res. (2018). doi:10.1002/jor.23893

23. Oliveira, P. G. F. P. de et al. Osseodensification outperforms conventional implant subtractive instrumentation: A study in sheep. Mater. Sci. Eng. C (2018). doi:10.1016/j. msec.2018.04.051

24. Oh, J. S. \& Kim, S. G. Clinical study of the relationship between implant stability measurements using Periotest and Osstell mentor and bone quality assessment. Oral Surg. Oral Med. Oral Pathol. Oral Radiol. 113, (2012).

25. Sennerby, L. \& Meredith, N. Implant stability measurements using resonance frequency analysis: Biological and biomechanical aspects and clinical implications. Periodontol. $200047,51-66$ (2008).

26. Mokhtari, M. R., Radvar, M., Sargolzaie, N. \& Moeintagavi, A. Resonance Frequency Analysis of Clinical Stability of Astra Tech and ITI Implant Systems. J. Periodontol. (2010).

27. Nedir, R., Bischof, M., Szmukler-Moncler, S., Bernard, J. P. \& Samson, J. Predicting osseointegration by means of implant primary stability. Clin. Oral Implants Res. (2004). doi:10.1111/j.1600-0501.2004.01059.x\rCLR1059 [pii]

28. Molly, L. Bone density and primary stability in implant therapy. Clin. Oral Implants Res. (2006). doi:10.1111/ j.1600-0501.2006.01356.x

29. Turkyilmaz, I., Sennerby, L., McGlumphy, E. A. \& Tözüm, T. F. Biomechanical aspects of primary implant stability: A human cadaver study. Clin. Implant Dent. Relat. Res. (2009). doi:10.1111/j.1708-8208.2008.00097.x

30. Esteves, T., Gaspar, J., Rua, J., Gaspar, R. \& João Mendes, J. Osseodensification for implant site preparation in the maxilla- a prospective study of 97 implants. Clin. Oral Implants Res. (2018). doi:10.1111/clr.48_13358

31. Lahens, B. et al. The effect of osseodensification drilling for endosteal implants with different surface treatments: A study in sheep. Journal of Biomedical Materials Research - Part B Applied Biomaterials (2018). doi:10.1002/ jbm.b.34154

32. Tian, J. H. et al. Alveolar Ridge Expansion: Comparison of Osseodensification and Conventional Osteotome Techniques. J. Craniofac. Surg. (2018). doi:10.1097/ SCS.0000000000004956

33. Khadtare, Y. \& Lulla, S. Osseodensification: An Inventive Approach in Implant Osteotomy Preparation Technique to Increase Bone Density. EC Dental Science (2018).

34. Kanathila, H. \& Pangi, A. An insight into the concept of osseodensification-enhancing the implant stability and success. Journal of Clinical and Diagnostic Research (2018). doi:10.7860/JCDR/2018/35626.11749

35. Slete, F. B., Olin, P. \& Prasad, H. Histomorphometric comparison of 3 osteotomy techniques. Implant Dent. (2018). doi:10.1097/ID.0000000000000767 